\title{
EFFECTS OF SIGNALISATION ON FOUR-ARM URBAN JUNCTION SAFETY
}

\author{
J. C. Golias \\ National Technical University of Athens, 5, Iroon Polytechniou, Zografou 15773, Greece
}

(Received 20 March 1995; in revised form 10 September 1996)

\begin{abstract}
This paper investigates the effect of signalisation on safety of urban priority controlled four-arm junctions. Forty-eight such junctions were carefully selected to have different values for the geometric and traffic characteristics that may influence the safety effects of signalisation. Long periods before and after signalisation were considered so that the effect of the regression to the mean phenomenon becomes negligible. Signalisation was found either to increase the junction safety or to leave it unchanged. Discriminant analysis showed that among numerous variables considered those significantly influencing the effects of signalisation on safety are the flow weighted average width per arm, the flow weighted average grade per stream, and a function of the product of the conflicting stream flows of the junction. The discriminant function developed predicts quite satisfactorily the behaviour of junction safety after signalisation. (C) 1997 Elsevier Science Ltd. All rights reserved
\end{abstract}

Keywords-Urban junctions, Traffic signals, Signalisation effects, Junction safety

\section{INTRODUCTION}

The installation of traffic signals at a priority controlled junction is based upon criteria which are basically related to traffic parameters (Federal Highway Administration, 1983). When the flows of conflicting traffic streams increase beyond certain ranges at a junction then the resulting delays increase to unacceptable ranges and at the same time the probability of an accident occurrence also increases significantly. The effect of the signalisation of the junction in these cases is to improve both traffic operation and safety.

Although there seems to be a consensus among relevant studies (Roess and Messer, 1986; Kimber and Hollis, 1979; Federal Highway Administration, 1986; Webster and Newby, 1964; Akcelic, 1988) that in cases of high traffic flows the signalisation of an intersection leads to the decrease of overall delay and the increase of overall capacity, no such agreement exists about the effect of signalisation on traffic safety.

Numerous studies on the effect of signalisation on junction safety have been carried out in the U.S.A. The earliest reported study on this subject (Vey, 1933) considered 599 junctions and found installation of traffic signals to result in a $20 \%$ overall decrease in accidents. On the other hand, research in Michigan (Solomon, 1959) has shown the total number of accidents at 39 rural junctions to increase an average $23 \%$ upon signalisation. A later study in Michigan
(Clyde, 1964) of 52 urban junctions also found an increase of $34 \%$ in total number of accidents. In California (Smith and Vostrez, 1964) the overall change after signalisation was a decrease of accidents by $39 \%$, in Detroit (Malo, 1967) a decrease of accidents by $47 \%$, while in Indiana (Schoene and Michael, 1968) and in Carolina (Cribbins and Walton, 1970) the overall change was an increase of accidents by $16 \%$ and $7 \%$, respectively. In a later California study (Hammer, 1970) new signal installation at 179 junctions was found to result in a $27 \%$ reduction in the total number of accidents, when a correction for the increase in exposure was applied. Accidents were found to increase by $24 \%$ after traffic signal installation in Virginia (King and Goldblatt, 1975), while they were found to decrease by $7 \%$ and by $2 \%$ in New York (New York State Department of Transportation, 1982) and in Milwaukee (Short et al., 1982), respectively.

A similar inconsistency in the results concerning changes in junction safety due to traffic signal installation was found in studies conducted outside the U.S.A. In Australia, a study of the total number of accidents before and after the installation of traffic signals revealed a significant reduction of $32 \%$ in accidents after signalisation (Andreassend, 1970). Teale (1984) examined 24 traffic signal installations in South Australia and an unspecified number in Western Australia; in both cases the overall accident 
number decreased by $20 \%$. In a more recent study in New Zealand (Frith and Harte, 1986) the total number of accidents after signalisation was found to decrease significantly at four-arm junctions. For three-arm junctions no significant change in the total number of accidents was found.

In Canada, the number of accidents at 13 junctions after the installation of traffic signals was found to decrease by $8 \%$ (Leckie, 1971). The effect of traffic signals on road accidents was also studied in Israel (Hakkert and Mahalel, 1978a) where it was found that at junctions with more than five accidents per year there is generally a reduction in the number of accidents, while at junctions with less than two accidents per year the number of accidents generally increases.

It is obvious from the above literature review that there is a serious lack of consensus on the overall safety impact of signal installation. This inconsistency is not surprising given that the safety effect of signal installation depends on a complex set of factors related to the geometric design of the intersection, the traffic characteristics, the accident history, etc. However, even in the studies that have similar values for these factors, the corresponding results do not seem to agree.

One important but often overlooked explanation for the fact that it is not possible to arrive at a well founded and commonly accepted conclusion on this subject is that there might have been practical limitations or methodological deficiencies in several of the above studies, on which current knowledge is based.

It should be noted that all studies reviewed above are of the 'before-and-after' type. The most common reason casting doubts on the validity of the results of these studies seems to be the failure to account for the regression to the mean phenomenon (Hauer and Persaud, 1983). According to this phenomenon even if the installation of traffic signals has no safety impact, junctions that recorded many accidents in the period before signalisation would, on average, record fewer accidents in the after period, while accidents would tend to increase at junctions that had few accidents. As a consequence, if as is usually the case, intersections are selected for signalisation on the basis of an unusually high accident record or on the basis of the safety warrant (Federal Highway Administration, 1986), a simple 'before-and-after' comparison is likely to overestimate the effect of the treatment.

The safety evaluation of traffic signal installation has also been considered in the context of studies of the cross-section type, where the results of crosssection analyses are used for inferences about the safety impact of adding a feature. However, the corresponding results are also inconsistent and as a consequence questionable. In one such study, signalised junctions with major and minor street annual average daily traffic of 10,000 and 8000 respectively were found (Syrek, 1955) to have higher accident rates than stop-controlled junctions with similar volumes. In a similar type of study (David and Norman, 1975) it was found that stop-controlled junctions had $30-60 \%$ fewer accidents than signalised junctions with similar volumes and the same number of approaches. Similar conclusions were drawn in another study (Hanna et al., 1976) where, for a given average daily traffic, signalised junctions were found to have a $29 \%$ higher accident rate than intersections with stop or yield control. However, a similar study in Australia (Thorpe, 1968) found that signalised and unsignalised junctions had similar accident rates. It is worth noting that this latter study used essentially the same data base which was used in the 'beforeand-after' study described earlier (Andreassend, 1970 ) and which led to the conclusion of a $32 \%$ reduction in accidents due to signalisation.

The main reason that cross-section studies such as those described above may have questionable findings is that the inference used about the safety impact of installing traffic signals overlooks the fact that the signalised junctions might have had a higher level of accident potential for various reasons not related to the installation of traffic signals. This higher accident potential is most probably the main cause for the installation of traffic signals and given that it is not taken into account in the cross-section studies it almost certainly underestimates the traffic signal installation safety effectiveness.

The above literature review was extensive and detailed in order to reveal in an indisputable way the rather embarrassing fact that there is very little substantial and well-founded knowledge about the safety impact of traffic signal installation. This is in agreement with the conclusions of a more recent work (Persaud, 1988) which at the end stresses the need for further research in the subject, using new methods of analytical to overcome the various analysis problems described above.

This work is an attempt towards this direction. More specifically the effect of traffic signal installation on the safety of four-arm urban junctions is investigated. The analysis is based on a 'before-and-after' accident comparison which takes into account the regression to the mean effect by considering long 'before' periods. An attempt is also made towards explaining the differences in the signalisation effect recorded among the junctions examined, on the basis of the geometric and traffic characteristics of each junction. 


\section{DATA COLLECTION}

Data were collected for periods before and after signalisation at 48 four-arm urban junctions in the greater Athens area of Greece. For each junction it was verified that when traffic signals were installed no other geometric or operational change was implemented. Furthemore, given that the scope of this research was to investigate the effect of signalisation on junction safety and more specifically whether this effect is influenced by the geometric and traffic characteristics, the junctions considered were carefully selected to have different values for the above characteristics varying within reasonable value ranges.

Due to the variety of the above characteristics at the junctions examined none of the standard methods for the elimination of the regression to the mean effects (Abbess et al., 1981; Hauer and Persaud, 1983; Persaud and Hauer, 1984; Hauer, 1986; Hauer and Lovell, 1986) was considered valid for this analysis. It was thus decided, as explained below, to consider long periods before signalisation so that the above effects become negligible (Abbess et al., 1981; Wright et al., 1988). In this context, for all junctions selected data were available for the seven consecutive years before signalisation. Furthermore, for reasons concerning reliability of the results, the 'after' period was at least 3 years long.

Data on accident occurrence at the 48 junctions finally selected were collected from police records. The number of accidents per year in the period before and after signalisation was recorded for each junction. It should be mentioned that only casualty accidents were considered, as reporting of these accidents is quite satisfactorily comprehensive in Athens area.

Traffic flow data were also collected from existing sources for the 'before' and 'after' periods at the above 48 junctions. The annual average daily traffic (AADT), for each year of the period considered, expressed in passenger car units (pcu), was finally used in the analysis for each traffic stream through each junction. Hourly traffic distribution factors as well as traffic growth factors of the study area were used to calculate the AADT of traffic streams throughout the particular study period at each junction when the required AADT measurements were not directly available.

Furthermore, on-site measurements at each junction gave information concerning the various geometric characteristics. Thus, for the major road arms of the junctions considered and for both traffic directions the number of lanes ranges from 2 to 6 , the total approach width ranges from $7.5 \mathrm{~m}$ to $21.5 \mathrm{~m}$ and the gradient from $+7 \%$ to $-6 \%$. For the minor road arms the range of the number of lanes is 2 to 4 , for the approach width $5.6 \mathrm{~m}$ to $12.5 \mathrm{~m}$, and for the gradient $+9.5 \%$ to $-8 \%$.

Although all junctions considered have four arms, the number of different traffic streams through each junction is obviously not the same, depending mainly on the number of existing one-way roads. This number was found to range from 6 to 12 in the junctions considered while the number of conflict points where the above traffic streams cross or merge was found to range from 8 to 24 .

At a considerable number of the junctions examined the major and minor road intersect almost perpendicularly. However, for all remaining junctions the angle at which the major and minor road intersect is significantly different from $90^{\circ}$ and ranges from $45^{\circ}$ to $140^{\circ}$.

Visibility along the major road arms from the minor road approaches was also measured at the junctions considered, and was found to range from $19 \mathrm{~m}$ to $86 \mathrm{~m}$ at a distance of $3 \mathrm{~m}$ from the stop line on the minor road arm.

As far as approach speed is concerned it should be mentioned that unfortunately there is no relevant information for the time period considered at each junction. Measurements carried out at an $80 \mathrm{~m}$ long section upstream from each junction revealed that at present the approach speed during the green phase ranges, among the junctions considered, from $40 \mathrm{~km} / \mathrm{h}$ to $75 \mathrm{~km} / \mathrm{h}$ on the major road and from $25 \mathrm{~km} / \mathrm{h}$ to $45 \mathrm{~km} / \mathrm{h}$ on the minor road during the morning off-peak period. Given that on the basis of each junction's area history there is no reason to expect speed changes of different scale among these junctions during the period examined, it is reasonable to assume that the present differences of approach speeds among the junctions reflect satisfactorily the corresponding differences during the period considered in this study.

It should also be noted that for the period considered in this study there were no counts for pedestrian flows. As it was thought that the intensity of pedestrian activity at a junction may influence driver behaviour and consequently accident occurrence, care was taken that it was of the same range at the junctions selected, assuming that this ensures that it was also of the same range during the period considered in this study. In this way it was ensured that any difference in the accident occurrence among the junctions is not due to pedestrian activity differences. Pedestrian activity intensity at each junction was expressed as the sum of pedestrian flow per hour crossing each of the four arms at the pedestrian crossing path or at an upstream distance not greater than $20 \mathrm{~m}$ from the corresponding crossing path. The above pedestrian activity intensity ranged from 50 to 
100 pedestrians per hour during the morning off-peak period at the 48 junctions considered.

\section{'BEFORE-AND-AFTER' ANALYSIS}

As already mentioned the 'before' period at all selected junctions was seven years long so that the regression to the mean effect becomes negligible and the true accident rate during this period is satisfactorily estimated. (Nicholson, 1987) The length of the 'after' period ranged from 3-5 years among the 48 selected junctions.

During the accident investigation period at each junction, which according to the above ranged from 10-12 years, no intervention concerning changes in the geometric layout or characteristics or the traffic regulation pattern was carried out at any of the selected junctions either before or after the installation of signalisation.

However, it is evident that during such long periods significant changes occurred in the flows of the various traffic streams at each junction. As a consequence, the safety of each junction was influenced by these changes (Hakkert and Mahalel, 1978b; Ward et al., 1983; Hauer et al., 1988) during the period under examination.

\section{Elimination of flow changes effects}

Given that the objective of this work is to investigate the effects of signalisation on junction safety it is obvious that the effects on safety due to traffic flow changes had to be eliminated. It has been shown (Brown, 1981; Ward et al., 1983; Hauer et al., 1988; Golias, 1988) that the expected number of accidents at a given junction, signalised or not, can be satisfactorily assumed to be a function of the incoming traffic stream vehicle flows.

More specifically, for priority controlled junctions it has been shown (Ward et al., 1983; Golias, 1992) that the total annual expected number of accidents at a junction is proportionate to an exposure index, which is the sum of a function of the products of the conflicting traffic stream flows, i.e. of the streams that cross or merge with each other. For the four-arm junctions under investigation in this work, the exposure index I considered to be the most suitable for the description of the effect of the flow on the accident potential of the junction is given by (Golias, 1992):

$$
I=\sum_{i=1}^{n}\left[Q_{i} \sum_{j=1}^{m_{i}} Q_{j}\right]^{0.5}
$$

where

$n$ : is the number of traffic streams entering the junction.
$Q_{i}$ : is the annual traffic flow of stream $i$ among the $n$ traffic streams entering the junction.

$m_{i}$ : is the number of traffic streams of the junction crossing or merging with stream $i$.

$Q_{j}$ : is the annual traffic flow of stream $j$ amongst the $m_{i}$ traffic streams crossing or merging with stream $i$.

It should be noted that the structure of the exposure index considers the conflict points of a traffic stream as a continuous string. This implies that when a driver is implementing a manoeuvre he assesses all crossing and merging streams together and proceeds under composite criteria in the completion of the manoeuvre.

Therefore, for the 'before' period during which the 48 junctions were operating as priority controlled junctions, the influence of the flow changes on the annual expected number of accidents was taken into account by considering that this number is proportionate to the above index.

It could be argued that sums of different functions of the products of the conflicting flows could have been used given on the one hand that such functions were shown to be less accurate but still satisfactory when used (Satterthwaite, 1981; Golias, 1992 ) and on the other hand that there is a variation in the geometric characteristics among the 48 junctions selected. However, it should be noted that as the purpose of this work is not to establish prediction formulae for the expected number of accidents but to investigate whether signalisation affects this number, only the percent change of the exposure index is in fact required in the analysis and not the exact form of the index.

The above change was investigated by considering two more exposure indices having the form of functions of products of the conflicting flows. These indices are the sum of products of the flows at each of the conflicting points (Hakkert and Mahalcl, $1978 \mathrm{~b}$ ) and the sum of the square root of the above products (Golias, 1992).

The difference of the annual percent change between the index finally used and the other two indices was found to be negligible. More specifically for the latter index it was less than $2 \%$ of the annual percent change of the index used, at all 48 junctions. For the former this difference was in the range of $4 \%$ at the vast majority of the 48 junctions while for a quite limited number of junctions the difference was higher, but never exceeding the value of $8 \%$.

The above results seem to indicate that at least for the period considered, the percent change of the expected number of accidents due to flow changes does not depend significantly on the exact form of 
the index, provided the index is a function of the product of the conflicting flows.

\section{Safety change analysis}

It is assumed, as is commonly done, that the annual number of accidents at a site follows the Poisson distribution. This is not only realistic given that the typical time and exposure conditions associated with the occurrence of accidents approximate closely the theoretical conditions that give rise to the Poisson distribution, but the assumption has also been shown to be valid (MacLean and Teale, 1982).

As a consequence, the number of accidents $X_{i}$ recorded during a year $i$ at a junction must be regarded not as the expected number of accidents at the junction, which of course cannot be measured, but as an estimate of this number. However, if a very large number of such annual estimates is available, then the mean of these estimates can be considered as the true annual expected number of accidents during the corresponding period.

It is for this reason that the 'before' period in the context of this study, was long enough so that the annual expected number of accidents could be evaluated with a satisfactory accuracy (Nicholson, 1987). More specifically, the 'before' period was 7 years long, a length that renders the above regression to the mean effects negligible (Abbess et al., 1981; Hauer, 1986; Wright et al., 1988).

However, as already mentioned, the effect of the annual traffic flow changes on the expected number of accidents had to be eliminated so that only the signalisation effect was investigated. The expected number of accidents $\lambda_{i}$ during a 'before' year $i$ at a junction with $X_{i}$ accidents during that year is assumed, as already mentioned, to be proportional to the exposure index $I_{i}$ for this year, given by equation (1).

The expected number of accidents in any period can then be estimated by the product of

(1) the ratio of the exposure during that period to the exposure during the before signalisation period;

(2) the observed number of accidents during the before signalisation period;

i.e. the expected number of accidents during an 'after' period consisting of $n$ years had the junction not been signalised would be:

$$
\lambda_{a}=\left(\sum_{i=1}^{7} X_{i}\right)\left[\left(\sum_{j=1}^{n} I_{j}\right) /\left(\sum_{i=1}^{7} I_{i}\right)\right]
$$

Taking into account the assumption that the annual number of accidents during a year of the 'after' period follows the Poisson distribution the number of accidents during the whole 'after' period also follows a Poisson distribution. Using the above equation for $\lambda_{a}$, a confidence interval for the actual number of accidents during this period had the junction not been signalised can be established on the basis of a chosen level of significance. If the number of accidents actually recorded in the period after signalisation falls within this confidence interval then the conclusion derived is that signalisation has no effect on the expected number of accidents at the junction. On the contrary a number of recorded accidents which is lower than the lower limit or higher than the upper limit of the confidence interval indicates an increase or a decrease of the junction safety correspondingly, i.e. a change in the expected number of accidents in both cases.

The calculation of the confidence interval described above, for each of the 48 junctions considered, took into account the uncertainty in the estimate of the corresponding Poisson parameter for the 'before' period by considering the confidence limits for this parameter (Nicholson, 1987). The level of significance used was equal to 0.10 . Comparison of these intervals with the corresponding number of accidents actually recorded during the period after signalisation showed that there was a decrease in the expected number of accidents at 28 junctions while for the remaining 20 junctions the assumption that there was no change in the junction safety could not be rejected at the level of significance used.

The above results seem to indicate that the effect of signalisation on junction safety is not uniform for all junctions. It could be argued that priority controlled junctions can be divided in two groups on the basis of whether signalisation increases junction safety or leaves it unchanged.

As far as accident type is concerned the 28 junctions belonging to the former group presented a significant increase in rear-end accidents from $21 \%$ on average before signalisation to $32 \%$ after it and a significant decrease in right-angle accidents from $44-28 \%$, respectively. The remaining 20 junctions presented also a significant decrease in right-angle accidents after signalisation from $36-22 \%$ on average but the corresponding increase in rear-end collisions from $37-42 \%$ was not significant. The changes in rear-end and right-angle accidents after signalisation in both junctions groups are offset by changes in the other accident types (head-on collision, pedestrian accident, etc.); however, none of these types was found to change significantly.

\section{SAFETY CHANGE FORECAST}

The existence of the above two groups when the cffccts of signalisation are considered, imposes the 
need for further analysis to investigate whether the classification of an uncontrolled junction as belonging to one of these groups is a random event or depends on certain parameters.

A considerable number of parameters that could influence, and therefore explain and forecast the effects of signalisation, was examined in the context of this analysis. All parameters considered are related to either the geometric or traffic characteristics of the junctions.

\section{Description of parameters investigated}

In this context the angle at which the two road axes of the junction intersect was considered. If the angle of intersection was different than $90^{\circ}$ the smaller angle was used. The angle of turn of the turning junction streams was also considered. However, given that the safety significance of this latter factor depends obviously on the traffic flow turning under this angle, the parameter used for each junction was the average angle for all the turning junction streams each weighted by the corresponding traffic flow during the 'before' period.

The grade of the various arms of each junction was considered in a similar way. The parameter finally used was the average grade for each stream of the junction weighted by the corresponding traffic flow. It should be mentioned that two grades were used for each stream, one for each arm used by the stream. The grade had a positive or negative sign depending on whether the traffic stream was moving downhill or uphill respectively.

The average pavement width of each junction arm weighted by the sum of the traffic flows of the streams passing through the arm was also investigated in relation to its influence on the safety effect of signalisation. This influence was also examined for the parameter that is derived by dividing the above average pavement width by the number of the junction streams.

The effect of visibility at a junction on the safety changes due to signalisation was also investigated. For obvious reasons only the visibility corresponding to traffic streams not having priority was considered. The parameter used in the analysis was the overall junction visibility, calculated as the average visibility for each such stream weighted by the product of the traffic flow of the two corresponding conflicting traffic movements, during the 'before' period. For cases where the traffic movement at a certain stream without priority was influenced by more than one other major traffic stream, and therefore more than one visibility corresponded to this traffic movement, the lower value was only used, weighted as above. The next higher visibility was also taken into account only if it was leading to a lower overall junction visibility. This approach emphasised the existence of unfavourable traffic conditions, as such conditions are expected to influence significantly the overall effect of signalisation on junction safety.

It was also thought useful to investigate whether the safety effects of signalisation depend on the traffic speed. The average speed at each approach during the green phase weighted by the traffic flow through the approach was used in this investigation. It should be remembered that the values of speeds used are those currently measured for each approach at an $80 \mathrm{~m}$ long section upstream from the junction and not those during the period examined, as there are no such speed measurements available. However, taking into account the flow changes at the junctions considered it is believed that the relative of the values of the above parameter for the 48 junctions had the actual speeds during the 'before' period been used are quite satisfactorily reflected in the relative values of this parameter as calculated in this analysis.

The number of traffic streams, the number of conflict points, the number of traffic lanes, and the mean number of lanes per stream were also considered in the analysis.

As far as parameters being more directly related to traffic flows is concerned, the mean entering traffic flow per arm, the mean traffic flow per stream, the exposure index I given in equation (1), and the exposure index I divided by the number of streams were considered in the context of this analysis.

Finally, it should be noted that a number of the parameters mentioned above were not only examined as separate isolated variables but were also used for the formation of more complicated variables. Thus, the combined influence of certain parameters was investigated for those cases that such a combination was meaningful and realistic, e.g. the product of grade and visibility, the product of angle of turn and grade, the ratio of visibility over speed etc. The variable value used for each junction was the average of the corresponding values of the appropriate junction elements (e.g. streams, arms, etc.) weighted by the appropriate traffic flows, in a way similar to that followed for the isolated variables.

\section{Parameter selection - discriminant analysis}

In order to select among the above parameters those with a statistically significant influence on the safety effects of signalisation and in order to quantify this influence, discriminant analysis was used.

Discriminant analysis (Cooley and Lohnes, 1971; Green and Rao, 1972) allows a statistically significant distinction among two or more mutually exclusive groups, in which a case may be allocated on the basis 
of a certain property. For the distinction among the groups discriminant analysis uses variables related to characteristics that are expected to differ in value among groups. The data required are the values of these variables for an adequate number of cases whose group membership is known.

Discriminant analysis allows the identification among a number of variables of those that are important for distinguishing among the groups. A procedure is also developed for predicting group membership for new cases on the basis of the values of the variables selected.

In discriminant analysis this is accomplished with the development of one or more linear combinations of the discriminant functions, which have the following form

$$
D_{i}=B_{o i}+B_{i 1} X_{1}+B_{i 2} X_{2}+\ldots+B_{i K} X_{K}
$$

where $D_{i}$ denotes the value of the discriminant function $i$, the $X \mathrm{~s}$ are the values of the $K$ independent variables, and the $B$ s are the coefficients estimated from the data.

In this work discriminant analysis is used for the development of a model that can predict the effects of signalisation of a priority controlled junction on safety. In this context two groups of junctions are considered: one including those junctions where safety increases when signalised and one including those where signalisation has no safety effect. By use of discriminant analysis a discriminant function is developed which can allocate a priority controlled junction to one of the two groups.

The analysis was carried out by using the SPSS Statistical Package (Norusis, 1988). The statistic used as the selection criterion is the Wilks' lambda (Tatsuoka, 1971). The stepwise selection procedure was followed, according to which the variables with the higher selection criterion values are entered successively, given that these values satisfy the entrance conditions set in relation to the criterion. It should be stressed that the variables finally included in the discriminant function through this procedure are selected from all variables considered on the basis of their statistical significance.

The criterion used as a measure of the effectiveness to distinguish among groups of the discriminant function developed is the percentage of cases classified correctly by applying the function to the database used.

The discriminant function developed after investigation of all the different variables considered is the following

$$
D=-2.017+0.491 W+0.278 G-1.284 I S
$$

where
$W:$ is the average pavement width per arm (in meters) weighted by the total traffic flow through the arm

$G$ : is the average grade per stream (in \%) weighted by the stream traffic flow

$I S:$ is the exposure index I given in equation (1) (flows in thousands of vehicles) divided by the number of junction streams.

According to the discriminant analysis results, junctions with discriminant function values lower than 0.116 are allocated to the group with junctions expected to have increased safety when signalised. On the contrary, for junctions with such values higher than 0.116 , no change in safety is expected due to signalisation, and these junctions are allocated to the other group.

The exact classification success of the discriminant function for the 48 junctions of the sample are given in Table 1. It can be seen that the overall classification success is in the range of $88 \%$.

The correlation between the discriminant function scores and the dsiscriminant variables was also examined by using SPSS (Norusis, 1988). It was found that the magnitude of this correlation is not in accordance with the magnitude of the corresponding coefficients of equation (2), as the rank of the variables on the basis of this correlation is from higher to lower $G, W, I S$. However, it was found that the sign of this correlation coincides with the sign of the corresponding coefficient, i.e. $W$ and $G$ are positively correlated with the discriminant function scores, while IS has a negative corresponding correlation. As a consequence, in attempting to interpret the kind of relation between the discriminant variables and the effects of signalisation on junction safety, the signs of the corresponding coefficients of equation (2) can be assessed without any problem.

In this context, the form of the discriminant function reveals that an increase in the average pavement width per arm weighted by the corresponding total arm traffic flow leads to an increased probability that the junction safety will not change due to signalisation. The above result seems to indicate that the

Table 1. Classification results of discriminant function

\begin{tabular}{lccc}
\hline & $\begin{array}{c}\text { Actual } \\
\text { Group }\end{array}$ & \multicolumn{2}{c}{ Predicted Group Membership } \\
\cline { 3 - 4 } & Allocation & Group 1 & Group 2 \\
\hline $\begin{array}{l}\text { Group 1: } \\
\text { Safety Increase }\end{array}$ & 28 & 24 & 4 \\
$\begin{array}{l}\text { Group 2: } \\
\begin{array}{l}\text { No safety Change } \\
\text { Overall Classification } \\
\text { Success: } 87.5 \%\end{array}\end{array}$ & 20 & $85.7 \%$ & $14.3 \%$ \\
\hline
\end{tabular}


improvement of traffic circulation conditions due to the increase of the average width of the corridor used by each vehicle passing through the junction diminishes the safety improvements due to junction signalisation.

A similar conclusion can be extracted for the average grade per stream weighted by the corresponding stream traffic flow, i.e. that an increase of this average grade diminishes the probability of safety improvement due to signalisation. It should be noted that, as already mentioned, downhill grades have a positive sign. As a consequence, an increase in the average grade, which in fact is the average grade of the course followed by each vehicle passing through the junction, means a more downward sloping direction of the traffic. Taking into account that the percentage of heavy vehicles in traffic for all junctions is less than $4 \%$, the increase of the downward sloping direction may be translated to better traffic circulation conditions at the junction. Therefore, the final conclusion seems to indicate that when a junction is signalised the expectation of safety improvement decreases as these conditions improve due to the grade increase.

As far as the third variable in the discriminant function is concerned, i.e. the exposure index divided by the number of junction streams, equation (2) reveals that as the value of this variable at a junction increases so does the expectation that signalisation of the junction will lead to safety improvement. It should be noted that the exposure index $I$ is a reflection of the existing complexity regarding the completion of all the different vehicle movements at the junction. As the value of the variable $I S$ increases, the traffic circulation conditions deteriorate and in this case signalisation seems to have an increased potential for safety improvement, according to the discriminant analysis results.

It can therefore be argued on the basis of the above results that the effects of junction signalisation increase for junctions that start out with poorer traffic conditions. The analysis revealed that the above three variables included in equation (2) can describe these conditions, reflecting adequately all other variables considered. Thus, for example the combined consideration of the average pavement width per arm weighted by the total traffic flow through the arm and of the exposure index I is believed to satisfactorily reflect traffic speed; similarly the use of the average pavement width per arm weighted by the corresponding traffic flow is believed to describe in a more comprehensive way traffic conditions than the number of lanes per arm, rendering the simultaneous use of this latter variable in the discriminant function unnecessary.

\section{CONCLUSIONS}

The effect of signalisation on junction safety was investigated at 48 urban priority controlled four-arm junctions. The 48 junctions were carefully selected to have different values within reasonable ranges for the various geometric and traffic characteristics that may influence the safety effects of signalisation.

Long periods before signalisation were considered at each junction so that the true accident rates during these periods could be satisfactorily estimated. The effects of traffic flow changes during these long periods as well as during the 'after' periods on junction safety were eliminated by relating the annual expected number of accidents at a junction to an exposure index. Thus, the signalisation effects on junction safety were isolated.

The 'before-and-after' analysis confirmed the conclusions of previous relevant studies that the signalisation of a priority controlled four-arm junction does not always have the same effect on safety. Thus, it may decrease the expected number of accidents at the junction but it may also leave the junction safety unchanged. Decrease of the junction safety due to signalisation was not observed at any of the 48 junctions investigated.

The possibility of predicting junction safety behaviour when the junction is signalised is obviously considered very useful. In this context, a considerable number of variables related to various geometric and traffic characteristics of the junction that could influence the safety effect of signalisation were examined.

The significance of this influence for each of the above variables was investigated by the use of discriminant analysis. A model was developed in the form of a discriminant function which can predict whether safety will increase or will not change if a junction is signalised. The variables that were found to have a statistically significant influence on the safety effect duc to signalisation and that were included in the model are the flow-weighted average pavement width per arm, the flow-weighted average grade per stream and the exposure index given by equation (1) divided by the number of junction streams.

Thorough examination of the discriminant function developed reveals that the more the values of the above variables ensure better conditions for the traffic through the junction, the smaller the probability that signalisation will increase junction safety. Therefore, the overall effect of signalisation on safety seems to be dependent on the overall quality of traffic circulation conditions, which are reflected by the combined action of the above three variables.

This conclusion could be an explanation, along with that of the regression to the mean effect, for the 
variety of conclusions about the safety effects of signalisation drawn in the various research works described in the introduction of this paper.

However, it is evident that the results of this work are only valid for the range of variable values of the sample considered. Further research is required to validate these results for more extreme variable values. It would also be very useful if similar models are developed for the prediction of signalisation effects on safety at priority controlled junctions with a number of arms other than four.

As far as changes in accident type are concerned there are indications that these too are dependent on the traffic conditions at the junction. However, these changes are also expected to be dependent on additional traffic and layout characteristics of the junction, the determination of which is believed to require further thorough investigation.

\section{REFERENCES}

Abbess, C., Jarrett, D. and Wright, C. C. (1981) Accidents at blackspots: estimating the effectiveness of remedial treatment, with special reference to the 'regression-tomean' effect. Traffic Engineering and Control 22, 9, $535-542$.

Akcelic, R. (1988) The highway capacity manual delay formula for signalised intersections. ITE Journal 3, 153-159.

Andreassend, D. C.(1970) Another look at traffic signals and accidents. In Proceedings of Australian Road Research Board, 6th Conference, Vol 5, Part 3, pp. 304-318.

Brown, J. R. (1981) A method for determining the accident potential of an intersection. Traffic Engineering and Control 22, 11, 648-651.

Clyde, M. N. (1964) Michigan study indicates signals increase accidents. Traffic Engineering 35, 2, 32.

Cooley, W. W. and Lohnes, P. (1971) Multivariate Data Analysis. Holt, Rinehart and Winston Inc., New York.

Cribbins, P. and Walton, J. (1970) Traffic signals and overhead flashes at rural intersections: their effectiveness in reducing accidents. Highway Research Record 325, 1-14, Washington, D.C.

David, N. A. and Norman, J. R. (1975) Motor vehicle accidents in relation to geometric and traffic features of highway intersections, FHWA. Department of Transportation, Washington, DC.

Federal Highway Administration (1983) Traffic control devices handbook. Department of Transportation, Washington D.C.

Federal Highway Administration (1986) Manual on Uniform Traffic Control Devices for Streets and Highways. Department of Transportation, Washington D.C.

Frith, W. J. and Harte, D. S. (1986) The safety implications of some control changes at urban intersections. Accident Analysis and Prevention 18, 3, 183-192.

Golias, J. C. (1988) Techniques for the control of the regression-to-mean effect in before-and-after accident studies. Technika Chronika, Scientific Journal of the Technical Chamber of Greece, Section A 8, 2, 8-26.

Golias, J. C. (1992) Establishing relationships between acci- dents and flows at urban priority road junctions. Accident Analysis and Prevention 24, 6, 689-694.

Green, P. E. and Rao, V. R. (1972) Applied Multidimensional Scaling. Holt, Rinehart and Winston Inc., New York.

Hakkert, A. S., Mahalel, D. (1978a) Effect of traffic signals on road accidents with special reference to the introduction of a blinking green phase. Traffic Engineering and Control 19(5), 212-215.

Hakkert, A. S., Mahalel, D. (1978b) Estimating the number of accidents at intersections from a knowledge of the traffic flows on the approaches. Accident Analysis and Prevention 10(1), 69-79.

Hammer, C. G. (1970) Evaluation of minor improvementspart 77. Traffic Signals. Traffic Development of California Division of Highways, CA.

Hanna, J. T., Flynn, T. E. and Tyler, W. L. (1976) Characteristics of intersection accidents in rural municipalities. Transportation Research Record 601, pp.79-82. TRB, Washington, D.C.

Hauer, E. (1986) On the estimation of the expected number of accidents. Accident Analysis and Prevention 18, 1, $1-12$.

Hauer, E. and Lovell, J. (1986) New directions for learning about the safety effects of measures. Transportation Research Record 1068, pp. 96-107. TRB, Washington D.C.

Hauer, E., Ng, J. C. N. and Lovell, J. (1988) Estimation of safety at signalised intersections. Transportation Research Record 1185, pp. 48-61. TRB, Washington D.C.

Hauer, E. and Persaud, B. N. A (1983) Common bias in before-and-after accident comparisons and its elimination. Transportation Research Record 905, pp. 164-174. TRB, Washington D.C.

Kimber, R. M. and Hollis, E. M. (1979) Traffic Queues and Delays at Road Junctions. TRRL Report 909, TRRL, Crowthorne, U.K.

King, G. F. and Goldblatt, R. (1975) Relationships of accident patterns to type of intersection control. Transportation Research Record 540, pp. 1-12. TRB, Washington D.C.

Leckie, D. A. (1971) Traffic signalisation: before and after accident analysis. Department of Highways, Ontario, Canada.

MacLean, A. S. and Teale, G. (1982) Probability distributions for traffic accidents, injuries and deaths. Australian Road Research 12, 1, 61-68.

Malo, A. F. (1967) Signal modernisation. Special Report 93, HRB, Washington D.C.

New York State Department of Transportation (1982) Effect of signalisation. Traffic and Safety Division, New York.

Nicholson, A. J. (1987) The estimation of accident rates and countermeasure effectiveness. Traffic Engineering and Control 28, 11, 518-523.

Norusis, M. J. (1988) Manual SPSS/PC. Advanced Statistics V2.0. SPSS Inc., Chicago.

Persaud, B. N. (1988) Do traffic signals affect safety? Some methodological issues. Transportation Research Record 1185 , pp. 37-47. TRB, Washington D.C.

Persaud, B. N. and Hauer, E. (1984) Comparison of two methods for debiasing before-and-after accident studies. Transportation Research Record 975, pp. 43-49. TRB, Washington D.C.

Roess, R. and Messer, C. (1986) The new highway capacity 
manual. Final Report, NCHRP Project 3-28B, Brooklyn, N.Y., Polytechnic University.

Satterthwaite, S. P. (1981) A survey of research into relationships between traffic accidents and traffic volumes. TRRL Report SR 692, TRRL, Crowthorne, U.K.

Schoene, G. W. and Michael, H. L. (1968) Effects on a change in the control device on intersection accidents. Engineering Reprint CE 237, Purdue University, Indiana.

Short, M. S., Woelfe, G. A. and Chang, C. J. (1982) Effects of traffic signal installation on accidents. Accident Analysis and Prevention 14, 2, 135-145.

Smith, W.C. and Vostrez, J. J. (1964) Evaluation of minor highway improvement projects. Highway Research News, 13, pp. 21-29. Washington D.C.

Solomon, D. (1959) Traffic signals and accidents in Michigan. Public Roads 30, 10, 234-237.

Syrek, D. (1955) Accident rates at intersections. Traffic Engineering 25, 8, 312-316.

Tatsuoka, M. M. (1971) Multivariate Analysis. John Wiley and Sons, New York.

Teale, G. (1984) The evaluation of the effectiveness of low cost traffic engineering projects. Report CR 22, Office of Road Safety, Department of Transport of Australia.

Thorpe, J. C. (1968) Accident rates at signalised intersections. Proceedings of the 4th Conference of Australian Road Research Board 4, 1, 995-1004.

Vey, A. H. (1933) Effect of signalisation on motor vehicle accident experience. In Proceedings of the Institute of Traffic Engineering, New York.

Ward, H., England, L., Murphy, R.S.D. and Moore, R. L. (1983) Accident occurence, traffic and road features at urhan priority type road junctions. Report to Accident Investigation Division, TRRL Contract No 842/539, TRRL, Crowthorne, U.K.

Webster, F. V. and Newby, R. F. (1964) Research into the relative merits of roundabouts and traffic signal controlled intersections. Journal of the Institution of Civil Engineers 27, 47-76.

Wright, C. C., Abbess, C. R. and Jarrett, D. F. (1988) Estimating the regression-to-mean effect associated with road accident black spot treatment: towards a more realistic approach. Accident Analysis and Prevention 20, 3, $199-214$. 